\title{
Transmission Performance of Flexible Relay-based Networks on The Purpose of Extending Network Coverage
}

\author{
Ardian Ulvan ${ }^{1}$ and Robert Bestak ${ }^{1}$ \\ ${ }^{1}$ Czech Technical University in Prague, Technicka 216627 Praha 6, Czech Republic \\ \{ulvana1, bestar1\}@fel.cvut.cz
}

\begin{abstract}
Wireless mobile terminal may performs as relay for some other terminals which require relaying assistance. The deployment of flexible relaybased wireless networks is expected to increase the capacity of the network when placed within the base station. It also extends the coverage when placed at the border of base station coverage. In this paper, the performance evaluation of transmission between mobile nodes at the border coverage is carried out. The CDMA-based network with frequency hopped and direct sequence was applied. The Erlang capacity is used as the main parameter. The results show, the relay nodes can extend the coverage, however the transmission performance have been limited by blocking probability and signal to noise ratio $\left(E_{b} / N_{0}\right)$. The results also show FH-CDMA has better performance than DS-CDMA.
\end{abstract}

Keywords: Mobile relays, transmission performance, transmission capacity

\section{Introduction}

A flexible relay-based wireless network is designed to extend the conventional point to multipoint communication. In addition, it also aimed to provide a broadband wireless access for the telecommunication users. The position of relays, where placed within the base station (BS) and at the border of BS's coverage, show different purposes. When relays are within the BS, they increase the capacity. Additionally, the relays extend the coverage when placed on the cell boundary [1].

In flexible relay-based system, beside a dedicated relay such as BS or access point (AP), the mobile wireless terminals in the network may perform as relays for some other terminals which require relaying assistance (mobile relays). The technology development to fulfill these characteristics is standardised by IEEE $802.16 \mathrm{j}$ which is still under progress.

In addition, since the family of IEEE 802.16 standards that based on OFDM is limited only to the air interface, it is also important to specify the network architecture [2]. The most common architecture is referred to Worldwide Interoperability for Microwave Access (WiMAX). In this paper we carried out some works in order to make the contribution for the implementations of flexible relay wireless OFDM-based networks that will improve the wireless broadband system of IEEE 802.16 families, yet in WiMAX network or others networks architecture. 
The work is focused on transmission performance of mobile hosts that act as relay. In our scenario, the mobile hosts are placed at the border of BS coverage, with a relay capability they support the connections to other hosts which are out of BS range. In the scenario, the CDMA-based network is deployed as it provides all parameters needed. The spread spectrum techniques of Frequency Hopping (FH-CDMA) and Direct Sequence (DS-CDMA) are taking into account in order to analyze the characteristics and the transmission capacity. The transmission performance of both techniques are simulated and determined by spreading factor, blocking probability and signal to noise ratio $\left(E_{b} / N_{0}\right)$.

The remainder of this paper is organised as follows. Section II gives brief description of flexible relay-based wireless networks scenario. Section III introduces our proposed scheme as well as all parameters that involved on the calculation. The performance analysis is given on section IV. As a final point, we present the conclusion in section $\mathrm{V}$.

\section{The Flexible Relay-based Network}

The work of flexible relay-based wireless network which was to distribute the high capacity available in the area surrounding the BS has been carried out in [3]. In this work we performed the ability of flexible relay in the purpose of increasing the networks capacity. The flexible relay means it could be fixed or mobile. When mobile station (MS) is connected to the base station (BS) it may act as a relay to other MSs who have limited or cannot be served by BS or out of BS's range. When a MS does not have relay capability, it acts as conventional subscriber station. Every node with relay capability acts as router and takes place to determine and maintain the routing to other nodes within the network. The relaying and routing functionality is seems as adhoc network. The connection scenario is shown on the Figure 1.

The uses of ad-hoc network as extended networks have been showed on previous works. Authors in [3] had determined the position of flexible relay within the BS. It was found that the flexible relay could increase the transmission capacity. The others works concluded that the uses of ad-hoc network have been increased the throughput capacity and power efficiency as well [4] [5]. On the other works, the use of CDMA on ad-hoc network has been considered. The implementation of spread spectrum on ad-hoc network shown some benefits such as better security, interference resistance, simultaneous co-located transmission and large scalability [6][7][8].

The important parameter of spread spectrum system is spreading factor or processing gain, which is defined as the transmission to information bandwidth ratio.

In FH-CDMA, the system can tolerate all interferences as long as the interfering signals are under the processing gain limit. Total bandwidth, $W$, is divided by $M$ orthogonal band frequencies (W/M). In this case, the purpose of processing gain is to reduce the interfering signals. When two or more transmitters have used the same band frequency, then the collision occurs. However, the collision can be recovered by all time coding. 


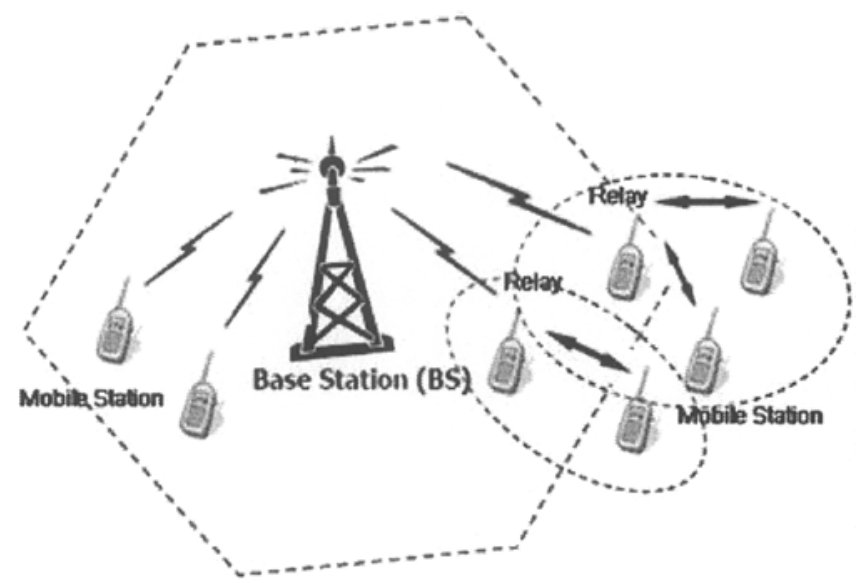

Fig. 1. Flexible relay-based wireless network. Some of the mobile hosts act as relay at cell boundary.

On the other hand, in DS-CDMA technique, the series of codes along the bandwidth, $W$, are multiplied by series of user data along the bandwidth, W/M. As a result is a series of signals which is transmitted along the bandwidth, $W$. The transmitted signal is spreading along the bandwidth which is $M$ times higher than original signal. In DS-CDMA, $M$ is known as spreading factor that analogous with processing gain in FH-CDMA. DS-CDMA uses spreading factor to reduce the minimum signal to interference noise ratio (SINR) at the receiver.

If the nominal requirement of SINR for FH-CDMA is $\beta$, therefore DS-CDMA reduces SINR to $\frac{\beta}{M}[7]$.

According to these characteristics, when they were deployed as a base system of flexible relay within the BS, FH-CDMA offers the interference avoidance. On the other hand, DS-CDMA is interference reduction [3]

In wide area wireless network with typical base station, the transmission intensity is defined by Erlang capacity. In CDMA system, the capacity is depend on the nominal value of interference. The blocking occurs when the interference power at reverse link reaches the given limit, which is assigned to maintain the quality of receiving signal [8]. Therefore, the users on CDMA system are allocated due to blocking probability. It is denoted as $\mathrm{B}_{\mathrm{CDMA}}$.

The call traffics in an individual time are random, as well as interference power. Due to these conditions, the blocking probability refers to call traffic distribution probability which is denoted as Erlang capacity. 


\section{System Scheme and Transmission Model}

As describe in [7], based on analysis of user's interference power in reverse link, the blocking probability can be defined. Let says $P_{m e d}=e^{m m_{d B}}$ with $\gamma=(\ln 10) / 10$, thus the blocking probability for DS-CDMA can be calculated by following equation:

$$
B_{D S-C D M A}=Q\left(\frac{\frac{W}{R_{b}}\left(1-\kappa_{0}\right)-\bar{M} \overline{v_{r}} P_{m e d} e^{\frac{1}{2} \gamma^{2} \sigma_{d B}^{2}}(1+\xi)}{\sqrt{\bar{M} \overline{v_{r}^{2}} P_{m e d}^{2} e^{2 \gamma^{2} \sigma_{d B}^{2}}\left(1+\xi^{\prime}\right)}}\right)
$$

The Erlang capacity is denoted by $\bar{M}$. The interference parameter, $k$, is comparative to cell loading, $x$, where $k=1-x$. If $k$ is changed into threshold value, $k_{0}$, then the loading threshold become $x_{0}=1-k_{0}$. Substitute this into (1) gives as follow:

$$
B_{D S-C D M A}=Q\left(\frac{\frac{W}{R_{b}} \chi_{0}-\bar{M} \overline{v_{r}} P_{m e d} e^{\frac{1}{2} \gamma^{2} \sigma_{d B}^{2}}(1+\xi)}{\sqrt{\bar{M} \overline{v_{r}^{2}} P_{m e d}^{2} e^{2 \gamma^{2} \sigma_{d B}^{2}}(1+\xi)}}\right)
$$

In case of FH-CDMA, the total interferences at BS occur only by users who have simultaneous transmitting on the same sub-channel, rather than all users. Therefore, the blocking probability is given as following:

$$
B_{F H-C D M A}=Q\left(\frac{\frac{W}{R_{b}}\left(1-\frac{\kappa_{0}}{M}\right)-\bar{M} \overline{\nu_{r} P_{m e d}} e^{\frac{1}{2} \gamma^{2} \sigma_{d B}^{2}}(1+\xi)}{\sqrt{\bar{M} \nu_{r}^{2} P_{m e d}^{2} e^{2 \gamma^{2} \sigma_{d B}^{2}}\left(1+\xi^{\prime}\right)}}\right)
$$

Similar to DS-CDMA, where Erlang capacity is denoted by $\vec{M}$, the interference parameter is $k$, the cell loading is $x$ and $k=1-x$. When $k$ is changed into threshold value, $k_{0}$, then the loading threshold become $x_{0}=1-k_{0}$. Then we substitute this into (3), the blocking probability can be calculated by: 


$$
B_{F H-C D M A}=Q\left(\frac{\frac{W}{R_{b}}\left(\frac{M-1+\chi_{0}}{M}\right)-\bar{M} \overline{\nu_{r}} P_{m e d} e^{\frac{1}{2} \gamma^{2} \sigma_{d B}^{2}}(1+\xi)}{\sqrt{\bar{M} \overline{v_{r}^{2}} P_{m e d}^{2} e^{2 \gamma^{2} \sigma_{d B}^{2}}\left(1+\xi^{\prime}\right)}}\right)
$$

As we can see from equations (2) and (4), the blocking probability equations can be denoted as $Q_{z}(z)$, where $Q_{z}(z)$ is defined as complementary cumulative probability distribution function (CCDF) from zero-mean and variant unit of standard random variables. The values of $\mathrm{Z}$ with associated to some values of blocking probability are available on a table of CCDF for standard normal distribution. The table is very advantageous in order to determine the Erlang capacity for the transmission channels.

To analyse the performance of proposed scheme, we also assume some parameters and variables as shown on table 1 .

Table 1. Parameters and variables

\begin{tabular}{|c|c|c|}
\hline Parameters/Variables & Symbol & Assumption \\
\hline Transmission Bandwidth & W & $1.2288 \mathrm{MHz}$ \\
\hline Data rate & $R_{b}$ & $9.6 \mathrm{kbps}$ \\
\hline Signal to noise ratio (median) & $m_{d B}=\frac{E_{b}}{N_{0}}$ & $5-7 \mathrm{~dB}$ \\
\hline Standard Deviation & $\sigma_{d B}$ & $2.5 \mathrm{~dB}$ \\
\hline Cell loading & $X_{0}$ & 0.9 \\
\hline Numbers of band freq. (spreading factor) & $M$ & $6-128$ \\
\hline Blocking Probability & $B_{C D M A}$ & $10^{-3}-10^{0}$ \\
\hline
\end{tabular}

\section{Performance Analysis and Results}

The transmission performance of relay-based networks on the purpose of extending network coverage can be shown on Figure 2, Figure 3 and Figure 4. The calculation and simulation are done for FH-CDMA and DS-CDMA.

From Figure 2, it can be shown that the Erlang capacity is increase when the blocking probability is getting higher. It simply means when a number of users transmission are blocked, several numbers of idle channels are available, as indicate by Erlang capacity value. However, the system has a critical section where Erlang capacity reaches the maximum value. It approximately reaches when blocking 
probability is 0,5 . Afterwards, the Erlang capacity decline gradually, even though the blocking probability is higher.

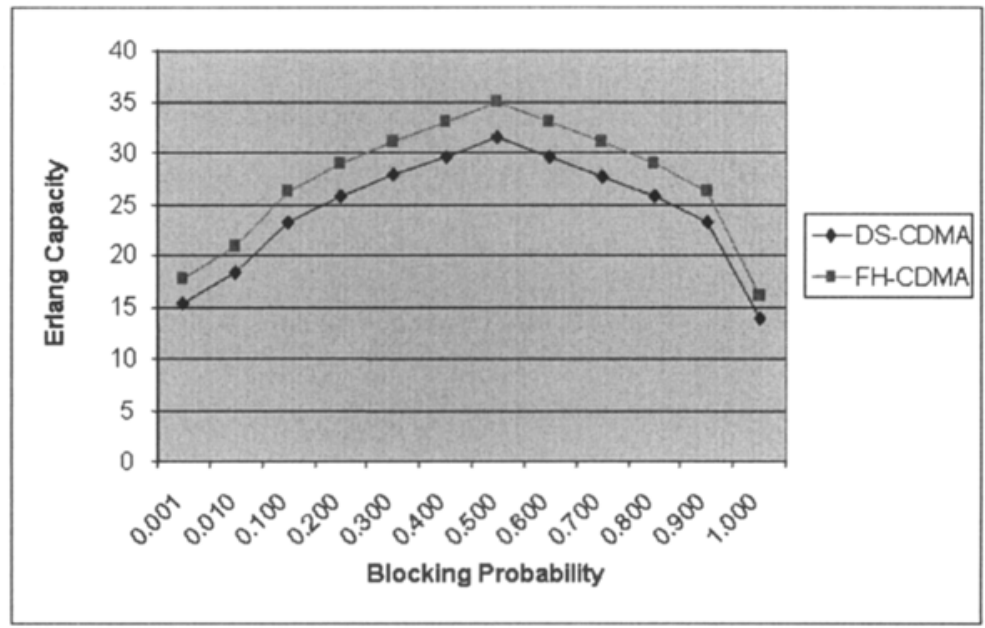

Fig. 2. The effect of blocking probability to Erlang capacity. FH-CDMA has better performance than DS-CDMA.

Furthermore as we can see from the graph, the values of FH-CDMA are higher than DS-CDMA. It can be explains that all interferences in DS-CDMA come from all active users. In contrast, all interferences in FH-CDMA come only from all active users using a same sub-band frequency.

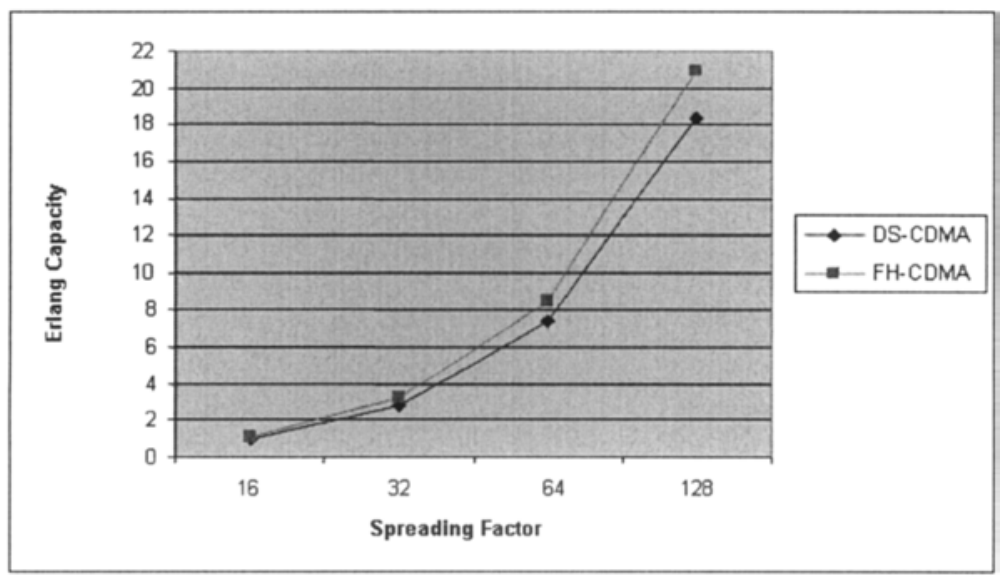

Fig. 3. The effect of spreading factor to Erlang capacity. FH-CDMA has better performance than DS-CDMA. 
The second aspect which influences the Erlang capacity is spreading factor, as shown on figure 3. As can be seen on Figure 3, the Erlang capacity is incline gradually by the inclining of spreading factor. FH-CDMA shows a bit better performance than DS-CDMA. The Erlang capacity has tremendous inclination when the spreading factor is 128 .

The last aspect that has been analysed to determine the transmission performance is the signal to noise ratio $\left(E_{b} / N_{0}\right)$. Figure 4 shows the effect of signal to noise ration $\left(E_{b} / N_{0}\right)$ to the Erlang capacity. As we can see from the graph, the Erlang capacity drop gradually while signal to noise ratio $\left(E_{b} / N_{0}\right)$ is increased. This circumstance quite similar to the effect of spreading factor when applied and analysed from relays within the $\mathrm{BS}$, as describe in [3].

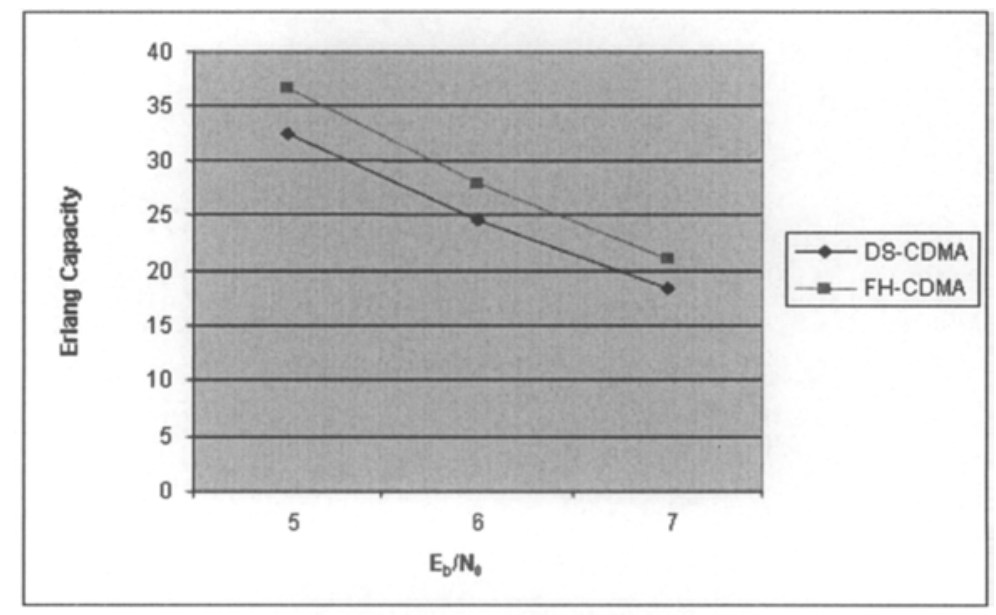

Fig. 4. The effect of $E_{b} / N_{0}$ to Erlang capacity. FH-CDMA has better performance than DSCDMA.

The signal to noise ratio $\left(E_{b} / N_{0}\right)$ expresses the signal's requirement power to get a successful transmission. In case of high interferences, the transmitter gains its signal power in order to reach better signal to noise ratio as well as to avoid the failed transmission. Unfortunately however, the gained power also increases significantly the probability of interference over the channel. It reduces the Erlang capacity. From the Figure 4 , we can also see that in term of signal to noise ratio $\left(E_{b} / N_{0}\right)$, the FHCDMA has better performance than DS-CDMA.

\section{Conclusions}

In this paper we analysed the flexible relay CDMA-based transmission performance for the purpose on extending coverage. We have illustrated that the MS at the cell boundary of BS coverage can act as a relay. It generally extends the cell or networks 
coverage. According to the results, there are two aspects that limit the transmission capacity for the relay, those are blocking probability and signal to noise ratio $\left(E_{b} / N_{0}\right)$. The blocking probability has certain value that match to maximum value of Erlang capacity. Based on simulation, the FH-CDMA shown better performance in all aspects rather than DS-CDMA.

Several aspects of this work deserve more analysis, especially the limitation use of band frequency on FH-CDMA. Another concern for future work is the implementation of $802.16 \mathrm{~d}, \mathrm{e}, \mathrm{j}$ OFDM-based flexible relay on WiMAX network as wireless broadband access system.

Acknowledgments. This work has been performed in the framework of FP6 project FIREWORKS IST-27675 STP, which is funded by European Community. The authors would like to acknowledge the contributions of their colleagues from FIREWORKS Consortium (http://fireworks.intranet.gr).

\section{References}

1. C. Hoynmann, et.al, "Flexible Relay Wireless OFDM-based Networks". Available at http://fireworks.intranet.gr. (2006).

2. IEEE Standard P802.16e/D8: "Part 16: Air Interface for Fixed and Mobile Broadband Wireless Access System", (2005).

3. A. Ulvan and R. Bestak, "Transmission Performance of Flexible Relay-based Networks". 2006. International Workshop on Digital Technology, Slovak Republic. (2006)

4. P. Lungaro, "Coverage and Capacity in Hybrid Multi Hop Ad Hoc Cellular Access System". Wireless@KTH, Radio Communication System Laboratory. 2003.

5. K. Holger, "Using ad-hoc extensions to cellular networks for capacity and energyefficiency improvements". Telecommunication Network Group. University of Berlin. Berlin. 2002.

6. W. Chunfeng, "An Introduction on Ad Hoc Network". Mita Lab, System Design Department, School of Science for Open and Environmental systems, Keio University. 2003

7. J. G.Andrews, X. Yang, A. Hasan, G. de Veciana, "The Flexibility of CDMA Mesh Networks". IEEE Wireless Communications Magazine. 2005.

8. P. Gupta and P.R. KUMAR,"The Capacity of Wireless Networks". IEEE Trans. Inform. Theory. vol. 46. No. 2, 2003. 